\title{
Literarische Termini in mitteliranischen manichäischen Texten $^{1}$
}

\author{
DESMOND DURKIN-MEISTERERNST (Berlin)
}

Mein Beitrag widmet sich den literarischen Termini in mitteliranischen manichäischen Texten und greift damit ein Thema auf, das über einen sehr langen Zeitraum ein zentrales Anliegen von WERNER SUNDERMANN gewesen ist. So greife ich zwangsläufig vieles von ihm auf. Die Termini habe ich in zwei Gruppen eingeteilt, die grob als Bezeichnungen von ganzen Texten und von solchen, die nur Textteile betreffen, sortiert werden können.

\section{Bezeichnungen von ganzen Texten}

Zunächst gilt es, den in mitteliranischen Texten belegten Bezeichnungen von Manis Werken nachzugehen. Einige davon sind schlicht Namen, andere stellen schon in sich literarische Termini dar; in einigen Fällen ist der Name zugleich ein literarischer Terminus geworden, das auffälligste Beispiel dafür ist 'Evangelium'.

\subsection{Evangelium (Evangelion)}

Der mittelpersische Text in M172 und M17 wurde von D. N. MACKENZIE in der Festschrift RUDOLPH 1994 mit Bezug auf die Übereinstimmung mit dem griechischen Text im Kölner Mani-Kodex herausgegeben. Offenbar verwendete Mani einige griechische Textbezeichnungen, was daran sichtbar wird, dass diese Bezeichnungen auch im Osten tradiert wurden und im Chinesischen Compendium belegt sind; für 'Evangelium' steht yinglun im chinesischen Text.

Mittelpersisch M172/I/V/10ff/ gwy̌sn 'y 'wnglywn zyndg 'y č́m 'wd gwš wcyhyd o'wd b'r 'y r'styy ncyhy(d)

'Das Sagen/Das Kapitel (?) des Lebendigen Evangeliums, das Auge und Ohr unterweist und den Ertrag der Wahrheit lehrt.'

1 Ich bedanke mich bei SUSANN RABUSKE für Korrekturen am deutschen Text. 
Das Evangelium als 'Buch':

Mittelpersisch M2/I/R/i/9-11/ps xwd'wn ${ }^{\circ}$ sh dbyr 'wnglywn ${ }^{\circ}$ ' $n y d w n b y g$ 'w 'd' prystyd o०

'Dann schickte der Herr drei Schreiber, ein Evangelion und zwei andere Bücher an Adda.'

Parthisch M5511/A/6/ [\{?\} \{rot\}'wn](g)l(yw)n jywndg (b.) $[=========$ $==\{?\}]$ (nicht veröffentlicht)

Parthisch in sogdischer Schrift TM406b/V/6/ 'st'w' $\delta$ 'wnklywn $z-y w($ ' $n t)$ 'kw 'Gepriesen ist das lebendige Evangelion.'

Aus mittelpersisch S1/V/6/ (')wnglywn ' $y$ wyst w: dw wdymwstyh'n 'Das Evangelion der zweiundzwanzig Wunder' sowie aus $\mathrm{S} 1 / \mathrm{V} / 4 /$ 'wnglywn ' $y$ ' $r \beta$ ncyhyd 'er lehrt das Araf (Alif) des Evangelions' und S1/V5/ 'wnglywn ' $y$ (t)ww ncyhyd 'Er lehrt das Tau des Evangelions' können wir sicher sein, dass es sich um das Evangelium in 22 Kapiteln nach den Buchstaben des manichäischen Alphabets (von Alif bis Tau) handelt, von dem auch An-Nadīm mit entsprechenden Angaben berichtet.

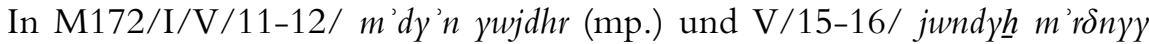
(sogd.) wird mit 'heiliges Buch' am ehesten auf das Evangelium Bezug genommen. Alternativ handelt es sich um eine Handschrift, in der neben dem Evangelium auch andere Texte versammelt waren.

M172 zeigt ferner eine interessante Kombination des mittelpersischen Textes mit einer sogdischen Paraphrase. Wir können nicht wissen, ob die sogdische Paraphrase eine parthische ersetzt hat. In der sogdischen Version wird ein parthischer Terminus als Äquivalent für einen mittelpersischen Terminus eingesetzt (sogd. = parth. wjy $\delta w^{\prime} \delta \delta$ für mp. w'xš ywjdhr 'heiliger Geist'; im sogd. Text folgt eine Ausdeutung mit zprt w'xšs 'id.', worin MACKENZIE 1994, S.

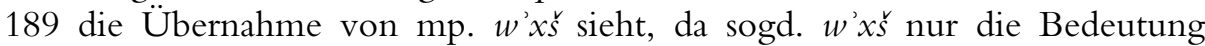
'Wort' hat), aber es besteht dennoch die Möglichkeit, dass es keine schriftliche parthische Form dieses auf Mani zurückgehenden Textes gegeben hat, weil für einen Sprecher des Parthischen die engverwandte Sprache Mittelpersisch weitgehend verständlich war und weil der mittelpersische „Original“-Text ein hohes Ansehen genoss. Dies scheint sich mit der Anfertigung sogdischer manichäischer Texte geändert zu haben, insofern nämlich, als eine Paraphrase angefertigt wurde, aber gerade die Beibehaltung des mittelpersischen Wortlauts zeigt das Ansehen des Originals. Dass eine gewisse Vertrautheit mit dem Mittelpersischen und dem Frühneupersischen unter den Händlern Zentralasiens bei der Wahrung des mittelpersischen Wortlauts eine Rolle gespielt haben kann, ist weniger wahrscheinlich, obwohl nicht geleugnet werden kann, dass der Text damit zugänglicher gewesen sein wird. 


\section{2 Šābuhragān ('das Schabuhr'sche [Buch]').}

Schon 1903/1904 fiel F. W. K. MÜLLER die Überschrift šbwhrg'n /šābuhragān/ auf einigen gut erhaltenen Seiten auf den von der ersten deutschen Turfanexpedition nach Berlin geschickten Textfragmenten auf (MÜLLER 1904). Er kannte den Titel aus An-Nadīms arabischem Bericht über den Manichäismus und wusste, dass es sich um einen Text handelte, den Mani dem Sasanidenkönig Šābuhr I (240-270/272) vorgelegt und den er auf Mittelpersisch geschrieben hatte. Laut An-Nadìm schrieb Mani alle seine anderen Werke auf Aramäisch. Dies lässt eine gewisse Diskrepanz zwischen dem westlichen und dem östlichen Kanon der Werke Manis aufkommen, denn das Šäbuhragān ist in keiner koptischen Quelle genannt. In Turfan ist das Šäbuhragān nur in seiner mittelpersischen Version zum Teil erhalten. Es scheint keine parthische Version zu existieren, was wohl für das hohe Ansehen des Textes spricht. Die zu vermutende Wertschätzung des Textes stimmt mit der sehr sorgfältigen Orthographie der erhaltenen Kopie überein, in der z.B. das auslautende $-\underline{h}$ häufig gesetzt wird und bei dem Pronomen 'wir' fast durchgehend ' $m \underline{h}$ statt ' $m$ ' $h$ geschrieben wird. Also können wir die Version aus Turfan wirklich als eine originalgetreue Abschrift eines Werkes Manis ansehen. Wenn der Kreis der Säbuhragān-Texte erweitert wird, d.h. um kosmogonische Texte (letzte Edition HUTTER 1992), die zwar nicht die Überschrift šbwhrg'n enthalten, aber im Inhalt dem von An-Nadìm angegebenen Inhalt des Werks nahe stehen, ändert sich die Beleglage in Turfan etwas, denn in der Berliner Turfansammlung gibt es neben mittelpersischen Fragmenten einen umfangreichen sogdischen kosmogonischen Text (M178), der auf einen solchen Text zurückgehen mag. Dies bedeutet wohl, dass, obwohl Manis mittelpersische Texte nicht ins Parthische übersetzt worden sind, eine sogdische Version sehr wohl hat angefertigt werden können.

Der volle mittelpersische Titel ist (in zwei Formen) $d w$ bwn (wzrg) ' $y$ šbwhrg'n 'die zwei (großen) Grundlagen/Prinzipien des Šäbuhragān'; es scheint, dass $d w$ bwn bzw. dw bwn wzrg allein auch der Titel des Werks gewesen sein kann. Die chinesische Bezeichnung von Manis Bildwerk da menheyi (MIKKELSEN 2006, S. 103) könnte nach BENVENISTE (s. HALOUN/HENNING 1952, S. 210) auf dō bungāhīg 'der zwei Fundamente' zurückgehen und somit vielleicht im Zusammenhang mit dem Šābuhragān stehen.

\subsection{Schatz des Lebens / Lebendiger Schatz}

Eine bemerkenswerte Bewahrung eines aramäischen Werktitels im Mittelpersischen hat HENNING in der Schreibung smty h' entdeckt. Er hat sie als eine korrumpierte Form von syr. simat hayyā $/ \bar{e}$ 'Schatz des Lebens' identifiziert (Haloun/HenNing 1952, S. 206-7; chin. xintihe MikKelsen 2006, S. 108). Das Werk ist ansonsten unter dem iranischen Titel mittelpersisch $n y^{\prime} n$ ' $y$ zyndg'n in M2 I R ii 34 und M2 I V i 14 bekannt; dort erfahren wir auch von 
einem Kapitel dieses Werks mit dem mittelpersischen Wort hrwbyšn 'Sammlung' in der Überschrift hrwbyšn ' $y$ dr'n 'das Sammeln der Tore' (M2 I R ii 35: $n y^{\prime} n$ ' $y$ zyndg'n hrwbyšn ' $y$ dr'n pyšy phypwrs ${ }^{\circ}$ 'Lies (ihr) 'Das Sammeln der Tore' des 'Schatz der Lebenden' vor!'); I V i 15 und 32-33 (hrwbyšn 'y pnz'n $\left.d r^{\prime} n\right)$, wo von Abschnitten über drei von insgesamt fünf Sinnesorganen die Rede ist: $d r^{\prime} y$ čsm' $n$; $d r^{\prime} y g w s^{\prime \prime} n$; $d r$ ' $y w y n y g$ 'das Tor der Augen; das Tor der Ohren; das Tor der Nase'). Es scheint bei hrwby̌̌n von dem ebenfalls belegten Verb hrwb- 'sammeln, zusammenbringen' trotz des Anklangs an einen buchtechnischen Terminus für eine Sammlung von Texten doch eher um das Beherrschen der Sinne zu gehen, denn die im Text gegebenen Beispiele gelten den Täuschungen und Fallen, in die ein Mensch durch seine Sinne geführt werden kann. Als parthische Bezeichnung dieses Texts können wir gnz erwarten, aber gnz ist im Parthischen nicht belegt; auch hier ist wohl keine parthische Übersetzung des mittelpersischen Textes bezeugt. Der griechische Titel $\theta \eta \sigma \alpha v \rho o ́ \varsigma$ ist auf den griechischen Bereich beschränkt und steht im Kontrast zu den Titeln 'Evangelion' und 'Pragmateia'.

Nebenbei bemerkt: Geringes aramäisches Material ist auch sonst im zentralasiatischen manichäischen Material vorhanden. Einen Teil eines aramäischen Textes hat YOSHIDA 1983 in M260 erkannt. YOSHIDA 1986 hat aramäisches Material auch in der chinesischen Hymnenrolle erfasst. In einem sehr beschädigten iranischen (parthischen) Fragment aus Turfan finden wir eine Überschrift mit dem Wort Qolasta ( $k w l^{\prime} s \underline{t}$ M481 R ii 5), das in der spätaramäischen Literatur vor allem als Bezeichnung einer mandäischen Textsammlung bekannt ist. Leider wissen wir nichts über den Inhalt des manichäischen Textes mit diesem Titel, der wohl nur 'Sammlung' bedeutet und auf keine bestimmte Textgattung hinweist. HenNING 1936, S. 12 hat den Beleg parthisch $\{\operatorname{rot}\}\left({ }^{\prime}\right) w s$ jyryft kwl'st 'und die Sammlung seiner Weisheit'? veröffentlicht.

\subsection{Giganten}

Das Buch der Giganten scheint allein mit dem Titel kw'n 'der Helden/Giganten' auszukommen: Im parthischen Text M5815/II/R/i/24-25/ heißt es: $w$ : $m n$ 'ny kw'n 'wd "rdhng 'ndr mrg kyrd o 'und ich machte ein anderes/zweites Kawān und ein Ārdhang in Marg (Merw).' Und im selben Text M5815/II/R/i/23-4/ 'wd k'w'n w: 'rdhng nyrd bwrd oo 'Und (er) brachte das Kawān und Ārdhang zu (mir).' In Überschriften würden wir eine vollständigere Angabe etwa mit dem Terminus 'Buch' oder 'Schrift' (nbyg) erwarten, aber keine solche Angabe hat sich erhalten oder ist zumindest bis jetzt erkannt worden. SunDERMANN hat 1994, S. 40 Anm. 1 auf das Fehlen einer parthischen Version des Gigantenbuchs hingewiesen. Allerdings hat MORANO 2009 Fragmente veröffentlicht, die parthische Zitate aus dem Gigantenbuch enthalten. 


\subsection{Hermas}

Mp M788/R/6/ (h)yrm' š(w)b'n 'der Hirte Hermas' ist ein Hinweis auf die Figur des Hermas, aber kein Beleg für das Vorhandensein eines Textes über Hermas in einer iranischen Sprache. Ein Text Manis wäre ein solches Werk nicht. HeNNING 1944, S. 142 Anm. 1 weist auf den Fehler 'Hirte Hermas' statt 'Hirte des Hermas' hin. Ein Grund hierfür könnte das Fehlen eines Textes über Hermas sein, d.h., der Schreiber hatte keine Möglichkeit, sich sonst über Hermas zu informieren. Allerdings weist mich W. SUNDERMANN zu Recht darauf hin, dass eine Deutung des Syntagmas als 'Hirte des Hermas' nicht ausgeschlossen sei.

\subsection{Henoch (hwnwx)}

$\mathrm{SIO} / 120 / \mathrm{I} / \mathrm{R} / 10 \mathrm{ff} /(s)[h m](y) z^{\prime} d$ gwpt $k w$ p $<$ h>ypwrs $d s[t]$ (nbyg 'y hwnwx 'zw)'.rg (...) [0... .... '] $\left.\left(y g{ }^{\prime} c\right) g w(y)^{\prime}\right)[n]$ (') $y(g)\left[m^{\prime} z y n d r^{\prime} n r^{\prime} y\right]$ 'Šahmizād sprach: 'Lies das Handschreiben Henochs, des «Schreibers, vor [...], das von der Rede, die [Dämonen betreffend, handelt]" (SUNDERMANN 1984b, S. 496-7). Für gwy̌́n ist statt 'Rede' vielleicht die Bedeutung 'Kapitel' anzusetzen. Die Bezeichnung dst nbyg 'Handschreiben' betont wohl den persönlichen Charakter des Textes 'hand(geschriebenes) Buch' oder 'persönlicher Bericht' [von Henochs persönlicher Reise ins Jenseits]. $\mathrm{Ob}$ das die reguläre mittelpersische Bezeichnung für das Henochbuch gewesen ist, lässt sich nicht feststellen. Auch hier handelt es sich nicht um ein Werk Manis.

\section{7 Ārdhang}

S. unter Šäbuhragān und Giganten oben. Ārdhang wird generell (An-Nadīm folgend) als Manis Bildbuch gedeutet. Aber SUNDERMANN 2005 hat Skepsis angemeldet und kommt zu dem Schluss, dass Ärdhang und Pragmateia dasselbe Werk bezeichneten und $\bar{A}$ rdhang die Bedeutung 'painstaking [Book]' habe. Ein

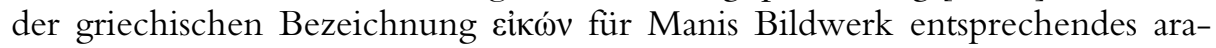
mäisches oder iranisches Wort wäre damit nicht direkt belegt, was aber nichts an dem gesicherten Vorhandensein des Bildwerks ändern würde. Das Feld manichäischer figurativer Kunst öffnet sich gerade durch die Forschungen J. EBERTS and Zs. GULÁCSIs, aber vor allem durch die Entdeckung manichäischer Bilder auf chinesischen Rollen in japanischen Sammlungen durch $\mathrm{T}$. MORIYASU und Y. YOSHIDA, s. MORIYASU 2011, YOSHIDA 2010.

\subsection{Buch der Mysterien}

Das im Chinesischen Compendium belegte Wort aluozan (HALOUN/HENNING 1952, S. 207; MiKKELSEN 2006, S. 102) deutet auf einen mittelpersischen Buchtitel r'z'n 'Geheimnisse, Mysterien' hin, der aber so nicht belegt ist. Die 
in koptischen und in griechischen Quellen belegte griechische Bezeichnung $\mu v \sigma \tau \eta$ prov wird demnach nicht im Osten beibehalten. Da das Syrische ebenfalls $r \bar{a} z \bar{a}$ (aus dem Iranischen entlehnt) kennt, spricht alles dafür, dass es sich um den ursprünglichen Titel des Werks handelt.

\subsection{Pragmateia}

Obwohl es nicht in einem iranischen Text belegt ist, bezeugt das bojiamodiye des Chinesischen Compendiums (HAloun/Henning 1952, S. 207; MiKKELSEN 2006, S. 102) die Verwendung dieses griechischen Titels auch bei den iranischen Manichäern. SUNDERMANN 2005 hat Überlegungen über den Inhalt dieses Textes veröffentlicht und dessen Gleichsetzung mit Ârdhang für wahrscheinlich gehalten. Er nimmt an, dass das Werk kosmogonische Legenden enthalten habe. Die Beibehaltung des griechischen Worts im Chinesischen Compendium spricht für dessen Verwendung durch Mani.

\subsection{Psalm(en)}

Wir kennen mehrere Bezeichnungen für Hymnen in der iranischsprachigen manichäischen Literatur. Sowohl Hymnenzyklen als auch einzelne Hymnen sind gut vertreten. Bemerkenswert ist die sehr große Zahl einzelner parthischer Hymnen in den Fragmenten der manichäischen Literatur. Die Vielzahl von Hymnen gilt gleichermaßen für die koptische wie für die iranische Literatur, aber die iranische Literatur setzt sich von der koptischen deutlich dadurch ab, dass die Hauptmasse der parthischen Hymnen alphabetische Hymnen sind. Ihren mesopotamischen Hintergrund zeigen sie auch in diesem Merkmal, das sie mit der mandäischen Hymnologie gemeinsam haben. Die Gemeinsamkeit geht über den reinen alphabetischen Strophenbau hinaus, denn in beiden Typen fängt eine nach dem letzten regulären Buchstaben gesetzte zusätzliche Strophe mit dem Buchstaben $\mathrm{n}$ an.

\subsection{Hymnenzyklus}

Gōwišn ì grīw zīndag (gōwišn ist 'Rede' = 'Aussprache' oder 'Kapitel' etwa als Teil einer Zeremonie?) s. unten.

Parthisch 'frywn, mittelpersisch 'fryn, chinesisch a-fu-yin

Bekanntlich heißen zwei lange parthische Verstexte qšwdg'n 'frywn und $w z r g$ 'n 'frywn. Es handelt sich bei ihnen um litaneiartige Lobgesänge mit wiederkehrenden Teilen (vgl. ähnliche koptische Texte im Psalm-book). Die Titel bedeuten entweder 'Lobpreisungen der Geringeren' und 'Lobpreisungen der Großen' oder die 'geringeren Lospreisungen' und die 'großen Lobpreisungen' mit Bezug auf ihren unterschiedlichen Umfang. Da sie z.T. auf einen aramäi- 
schen Text von Mani zurückgehen können, hat M. BOYCE einen Zusammenhang mit der Bezeichnung 'Psalmen' in den koptischen Listen hergestellt und die Texte als „Mani’s Psalms“ charakterisiert. E. MORANO und ich haben diese Bezeichnung beibehalten, obwohl damit nicht gesagt werden soll, dass es sich wirklich um Texte handelt, die direkt oder indirekt in ihrer bezeugten iranischen Form auf Mani zurückgehen (s. DURKIN-MEISTERERNST/MORANO 2010). Wenn man eine Autorschaft Manis annehmen möchte, so würde man wiederum erwarten, dass die mittelpersische Version des Textes auf Mani zurückgeht; eine parthische Version wäre auf jeden Fall sekundär und eine sogdische Version von der parthischen oder der mittelpersischen abhängig. N. SiMS-WILLIAMS hat uns ausdrücklich auf die letzte Möglichkeit aufmerksam gemacht und bestätigt einen Hinweis, den wir im Text gefunden haben (am Anfang des wzrg'n 'frywn, wo der parthische Text einen Plural 'ymyn 'diese' hat, hat der sogdische Text den Singular ' $y n$ ' $k$ 'dies'; für den mittelpersischen Text lässt sich ein Singular * $y n$ vermuten). Ein Teil des Textes lässt sich als ein Text Manis identifizieren: Im Mittelpersischen steht $y s n$ mit Bezug auf den Text; im Parthischen und im Sogdischen stehen dafür "frywn und "prywnh.

I. GARDNER hat zum Glück noch vor der Drucklegung unserer Edition gesehen, dass das wohl zwischen den zwei "frywn-Texten stehende Gebet nm'c br'm 'wd 'fryn'm ein sehr hohes Alter hat und sowohl in einer griechischen Version aus Ägypten Eủxخे $\tau \tilde{\omega} v \pi_{\rho \circ} \beta 0 \lambda \tilde{\omega} v$ (P. Kellis Gr. 98) als auch z.T. bei An-Nadìm überliefert ist. Der griechische Text hat in der Überschrift den Terminus ev̉xๆ́; ein separater Titel für den parthischen Text ist nicht bekannt. (s. jetzt GARDNER 2011).

\subsection{Namen/Titel statt Bezeichnungen}

Die zwei großen parthischen Hymnenzyklen, von WALDSCHMIDT-LENTZ 'Gliedhymnen' genannt, da das parth. Wort hnd' $m$ 'Glied' in den Überschriften und Rubriken steht, haben Namen, die aus dem ersten Wort Huyadagmān oder aus den ersten zwei Wörtern Angad Rō̌snān gebildet sind. Dieses Phänomen ist auch sonst in der manichäischen Literatur (z.B. 'ymyn 'hynd in Psalmenüberschriften für nur einen Teil des Textes des $w z r g ' n$ 'frywn) und ebenfalls in der awestischen Literatur bezeugt. Huyadagmān heißt 'glücklich wir' im Sinne von 'Glück für uns'. Interessant ist, dass der Titel angad rō̌snān, für sich genommen, keinen rechten Sinn ergibt: Er bedeutet eigentlich ein unvollständiges 'gekommen ist der Lichter', während im Text erst das folgende Wort friyānag 'Freund' den Sinn 'gekommen ist der Freund der Lichter' oder 'gekommen ist der Geliebte der Lichter' ergibt.

Bedeutsam ist auch hier, dass es von einem Teil, dem Anfang des Huyadagmān, eine sogdische Version gibt. Zusammen mit der sogdischen Version des wrzrg'n 'frywn macht das fast die ganze belegte sogdische Hymnologie aus. Sonst sind nur sogdische Abschriften mittelpersischer und parthischer Hymnen sowie sogdisch geprägte Kantillierungen belegt - beides Phänomene, die uns 
zeigen, dass Sogder oder/und sogdisch-sprachige Uiguren die mittelpersischen und die parthischen Hymnen so vortrugen.

\subsection{Bezeichnungen einzelner Hymnen von unterschiedlicher Länge}

Mittelpersisch $m h r\left(<\star_{m a n} \theta r a-\right)$. Häufig in Überschriften und Rubriken.

M1 'yn mhrn'mg 'dieses mahr-Buch' als Bezeichnung des Textes, aber wohl auch des Buchs (das wohlgemerkt keineswegs nur mittelpersische Hymnen auflistet).

Parthisch $b^{\prime} s^{\prime} \underline{h}$ (auch ein Verb $b \bar{a} s_{-}$' 'singen', ebenfalls sogd. $p$ 'š v.); sogd. $p$ 'šyk ein substantiviertes Adjektiv, SimS-WILliams 1983, S. 137. Diese Termini sind ebenfalls häufig in Überschriften und Rubriken. Die Tatsache, dass es sich bei parthisch $b^{\prime} s^{\prime} \underline{h}$ um ein indisches Lehnwort handelt, bedeutet keinen prinzipiellen Unterschied zu mittelpersischen Hymnen. RECK 2004, S. 42ff. geht auf Hymnen ein.

Mittelpersisch 'pwryšn. Für BOYCE 1968, S. 74 ist die Bezeichnung 'pwry̌̌n die mittelpersische Entsprechung zu parthisch 'frywn; da es allerdings auch mittelpersisch 'fryn gibt, ist diese Übereinstimmung nicht vollkommen. Für BOYCE stellen 'pwry̌̌n- und 'frywn-Texte lange ungeteilte Verstexte dar. Die wiederholten Anreden einer oder mehrerer Gottheiten können einen litaneiartigen Charakter haben.

In Rubriken, die Angaben zu der Melodie eines Hymnus machen, kann der Hymnus mit einem Demonstrativpronomen gekennzeichnet werden, z.B.

\begin{tabular}{|c|c|c|}
\hline $\begin{array}{l}\text { p. } \\
\text { p. }\end{array}$ & $\begin{array}{l}\text { M1367/R/1/ } \\
\text { RH(725) M798b/B/6/ } \\
\text { M31/II/R/6/ } \\
\text { HLS(1391) M5751/A/i/7/ } \\
\text { BBB(446) } \\
\text { RH(796) M341a/B/5/ }\end{array}$ & $\begin{array}{l}\text { (') yn yk pd k[..]ny nw'g } \\
\text { 'yn sh [pd y]q nw'g } \\
\{\text { rot } \text { pd whmn 'wzxt nw'g } \\
\text { pd b'm br'z'g nw'(g) } \\
\text { 'yn pd by hy 'bz'r nw'k } \\
{[\text { mwn]w } 1 \text { 'jgnd (')[gd] ['] }(c) \text { whyst }}\end{array}$ \\
\hline
\end{tabular}

Ein explizites Nomen für den Hymnus scheint hier nicht vorzukommen.

q'dwš

Eine Anzahl von kurzen Huldigungsstrophen, die mit einem bis vier $q$ 'dws 'sanctus' (einem hebräischen Lehnwort) anfangen und wohl in Litaneien Verwendung fanden, ist belegt. Möglicherweise sind zumindest einige von diesen Texten Refrains.

RECK (2004, S. 45ff.) geht auf „Kurzhymnen“ ein und vergleicht diese mit syrischen Kurzhymnen mit der Bezeichnung 'enyānē. Wenn Rubriken auf die mitteliranischen Kurzhymnen hinweisen, werden die sonst üblichen Bezeichnungen für „Hymnen“ verwendet. Möglicherweise haben auch Gebete (parth./mp. $n m^{\prime} c$, sogd. $n m^{\prime} c y w$ ) als Hymnen bzw. Kurzhymnen gegolten. $\mathrm{Zu}$ sogd. ymkw' 'ty 'Brywnh KG 468, s. unten.

$\mathrm{Zu}$ Refrains, s. unten bei Textbestandteilen. 


\subsection{Briefe}

Der erhaltene Teil des „Bet- und Beichtbuchs“ (M801a) fängt mit dem Ende eines (in der Überschrift und in der Schlussrubrik) als mwhr dyb 'Siegelbrief' bezeichneten mittelpersischen Textes an. Der Text ist eindeutig ein Grußschreiben von Mani an seine Gemeinde, und selbst im sogdischen Text wird der mittelpersische Wortlaut beibehalten. Das Wort deb ist ebenfalls im Chinesischen Compendium belegt (HALOUN/HENNING 1952, S. 207), und einzelne Briefe sind in einem sogdischen Fragment M915 (HALOUN/HENNING 1952, S. 206) angegeben: hier wird die sogdische Bezeichnung frurtyy verwendet, z.B.: [pr] sysn ptyy frwrty $(y)$ [w'nw] npyst 'im Sīsin-Patī-Brief schreibt er wie folgt: [...]'. Mittelpersisch prurdg kommt auch vor. Manis Briefe vervollständigen sein Werk, sind aber auch eine bewusste Aufnahme der paulinischen Epistolographie (SUNDERMANN 1984a, S. 239) und so in der Bezeichnung auf syr. eggertā zurückzuführen. Die wichtigsten Briefe sind bei An-Nadīm aufgelistet.

\subsection{Sermon}

Für 'Sermon' ist parthisches wyfr's, aber auch einfach sxwn 'Rede' belegt.

SUNDERMANN 1986, S. 67 setzt für sxwn 'Kapitel einer Lehrschrift, Homilie, Parabel, Versrede' und für wyfr's 'Homilie, Traktat, Versrede' an. Für saxwan stellt SUNDERMANN 1984a, S. 229-230 auf: 1. Kapitel von Lehrschriften Manis: 'Kapitel'; 2. Traktat oder Homilie; 3. in der Seitenüberschrift einer Parabelsammlung; 4. Versede. ZorMP saxwan im Texttitel sūr saxwan 'Rede vom Mahl' ist kein Vorbild für den manichäischen Gebrauch gewesen. Mittelpersisch gōwišn und parthisch saxwan übersetzen syrisch mēmrā 'Rede $\sim$ Versrede' (ebendort S. 230). Für wyfr's weist SUNDERMANN 1984a, S. 232 auf das Verb wifrăs- 'lehren, verkünden' hin und stellt fest, dass es „eigentlich die mündliche Predigt darstellte“. Es bezeichnet: 1. „vorwiegend Abhandlungen vom Charakter eines Traktats oder einer Homilie“; 2. „die Versrede“ (ebd., S. 233). SUNDERMANN fasst diese Bedeutungen unter Bezeichnung "literarische Homilie' zusammen. Er hält eine Entstehung der Bezeichnung in der höfischen parthischen Dichtung für möglich (S. 236); aber die manichäische Verwendung wird auch hier eine syrische Vorlage haben, möglicherweise tahwitita 'Darlegung, Unterweisung, Homilie'. In den erhaltenen Überschriften zum parthischen Sermon von der Seele ist nur wyfr's belegt: gy'n wyfr's = 'br hry 'yr wyfr's (SUNDERMANN 1997, S. 55) [in sogdischer Schrift ky'n wBr's]. Für den parthischen Sermon vom Licht-Nous sind wyfr's und sxwn gleichgesetzt: mnwhmyd rwšn wyfr's = mnwhmyd rwšn sxwn (SUNDERMANN 1992, S. 44).

Hier geht es um umfangreiche Texte, aber $w y f r$ 's scheint auch für kürzere Texte belegt zu sein: 'rdhng wyfr's und hwnsndyft wyfr's, von denen jeweils nur eine Seite erhalten ist, so dass der Umfang nicht ermittelt werden kann. Jedenfalls haben die letzten zwei Texte nicht die recht umfangreiche Beleglage der zwei erstgenannten Texte. Weitere Belege sind: $n^{\prime} g^{\prime} n$ wyfr's; r'h'n wyfr's; 
d'rwbdgyftyg wyfr's; prnybr'nyg wyfr's. In einem fortlaufenden Text ist bg'nyg wyfr's MKG(1312) belegt, für das SUNDERMANN die Übersetzung 'göttliche Belehrung' gewählt hat. Als Teil eines Titels scheint wyfr's nicht nur 'Erörterung', sondern auch 'Kommentar' zu bedeuten. Die Übersetzung 'Sermon' ist ungenau und bezieht sich auf die Gleichsetzung der langen Erörterungen in den Texten gy'n wyfr's und mnwhmyd rwšn wyfr's mit Homilien bzw. Sermonen. Allerdings sind diese Texte nicht wirklich diskursiv, sondern eher Lehrtexte, die sehr viele Einzelheiten und vor allem strukturierte Listen enthalten. Es gibt durchaus Berührungspunkte mit den koptischen Homilien und Kephalaia-Texten sowie mit mittelpersischen und mit parthischen Homilien, aber der strenge Aufbau ist unübersehbar.

Das sogdische $w \gamma \delta \beta^{\prime} \gamma$ 'Diskurs, Darlegung' (+ $c n$ 'über') (SUNDERMANN 1984a, S. 233: wrtl. 'Die Entfaltung') könnte zu beiden Bedeutungen 'Sermon' und 'Kommentar' passen, aber $w \gamma \delta \beta$ ' $\gamma c$ 'Prediger' bestätigt eher die erste Mög-

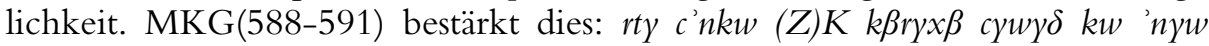

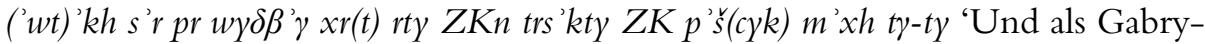
$\mathrm{ab}$ von dort in eine andere Gegend ging, um zu predigen, da war der Christen Fastenmonat gekommen.' (SUNDERMANN 1981, S. 49).

SUNDERMANN 1986, S. 68 setzt für $w \gamma \delta \beta$ ' $\gamma$ 'Kapitel einer Lehrschrift, Homilie, Traktat, Versrede' an, räumt aber auch die Bedeutung 'Verkündigung' ein. SUNDERMANN 1984a, S. 233ff. stellt fest, dass das Wort auch in christlichen und in buddhistischen Texten verwendet werde und „daher gewiß keine manichäische Neuprägung“" sei.

Ein ähnliches sogdisches Wort ist $p r \beta$ ' $r$ 'Erklärung, Verkündung, Lehre'.

SUNDERMANN ging 1992 der Frage nach, ob die iranische Literatur Texte wie die koptischen Kephalaia-Texte gekannt hat. Trotz der Unterschiede zwischen den koptischen und den mitteliranischen Fragmenten kommt er zu dem Schluss (SUNDERMANN 1992, S. 316), dass es eine ,frühmanichäische Sammlung von Lehrreden Manis vom Charakter der Berliner koptischen Kephalaia ['Die Kephalaia des Lehrers'] in der Überlieferung seiner Jünger gegeben hat, die sich in west- und ostmanichäischer Tradition nachweisen läßt". In einem Aufsatz aus dem Jahre 1975 untersuchte er, ob Yimki-Homilien vorkommen, d.h. Homilien aus Anlass der Yimki-Fastentage. Im letzteren Fall ist gwy̌̌n in Überschriften als Bezeichnung von Homilien belegt. Dort ist von der stattlichen Anzahl von 45 Homilien ( $($ wwy̌̌n'n $n$ ) die Rede. 


\section{Anmerkung}

Ein Werk mit dem Titel išnōhr, das man unter der Bezeichnung „The išnōhr texts“ als ein von HeNNING in Vorbereitung befindliches Buch im „Catalogue" von M. BOYCE 1960 findet, gibt es nicht. HENNING bezog sich auf das häufige Vorkommen des Wortes 'šnwhr in Texten, die SUNDERMANN 1997 als Teile des Sermons von der Seele ( $g y^{\prime} n$ wyfr's) 1997 veröffentlicht hat. Ein Werktitel, der das Wort '̌nnwhr enthält, ist nicht belegt.

\subsection{Buch der Erzählungen/Gleichnisse/Parabeln}

$\bar{a} z a n d, \bar{a} z e n d, \bar{a} z a n d n \bar{a} m a g$, s. unten

\section{$1.17 X w^{\prime} s t w^{\prime} n y \beta t$}

$X$ 'stw'nyft 'Bekenntnis' als Text ist bekanntlich nur in einer türkischen Version vollständig erhalten, aber HENNING 1940 und SiMS-WILLIAMS 1991 haben Teile einer sogdischen Version veröffentlicht. Der Titel (mit dem parthischen Suffix $-\gamma f t$ ) und einige weitere Merkmale des Textes deuten auf eine parthische Vorlage hin; eine sogdische Vermittlung ist wahrscheinlich, ohne dass sie auf eine sogdische Version von gleichem Umfang schließen ließe.

\subsection{Ysn 'Verehrung'}

z.B. in einem Paar oder einer Dreierreihe mit anderen Termini:

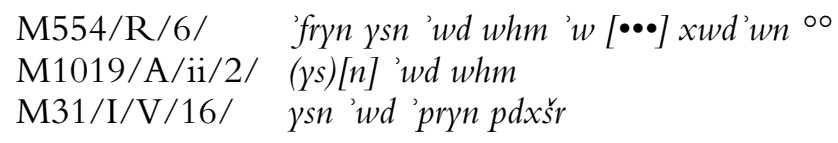

Bezeichnet das Wort eine Textgattung oder vielleicht eher eine Handlung? Das Wort $y$ sn stammt aus dem zoroastrischen Bereich (s. SUNDERMANN 2004). Bedeutsam ist sicher, dass es nur in mittelpersischen Texten verwendet wird. Es geht also um eine bewusste Übernahme von Schlüsselbegriffen der mittelpersischen zoroastrischen Literatur in die manichäische Literatur. Dies ist gut sichtbar in:

M379b/A/9ff/ ['yn 'st] ysn 'y y yst m(r) [ym'ny] [prystg ]'y y yšw' 'r(y)['m'n] [pd $\left.k^{\prime} m\right](y) s_{s}^{\prime} y^{\prime} y$ by $z r w\left(^{\prime}\right)\left[n k^{\prime}\right]\left[{ }^{\prime} w\right](w) z r g y \underline{h}{ }^{\circ} w: n(h)[w](p)\left[t^{\prime} n\right]\left[r^{\prime} z^{\prime}\right](n) ' b h w p t^{\circ}$

'This is the yasn ('worship') that Lord Mani the apostle of Jesus Aryaman offered by the will of God Zurwān [when] he revealed the greatness and the concealed secrets.' (DURKIN-MEISTERERNST/MORANO 2010, S. 117). 
Auch $y y^{2} w^{\prime}$ ' $r y^{\prime} m$ 'n ist zoroastrisch motiviert (s. Müller 1904, S. 28). Die parthische und die sogdische Version dieses Textes verwenden beide äfrīwan, parth. 'frywn, sogd. "prywnh. Somit scheint zumindest an dieser Stelle ein Text oder ein Textteil gemeint zu sein.

\subsection{Bezeichnungen nichtmanichäischer Texte}

Neben der bewussten Verwendung des mittelpersischen Wortes ysn mit unverkennbarem awestischen Hintergrund hat W. SUNDERMANN auf eine Reihe zoroastrischer Bezüge im Sermon von der Seele hingewiesen (SUNDERMANN 1997, S. $103(\$ 14,3), 105(\$ 18,3), 110(\$ 22,2), 113(\$ 32,1), 114(\$ 32,2), 119$ $(\$ 46,1), 122(\$ 65,1))$. Dort ist nsk (GW \32, \46, vielleicht als $n s g \ 21$ und ergänzbar in $\$ 65) die Bezeichnung awestischer 'Bücher' sowie die Namen der awestischen Texte 'whnwyt g'h (für aw. Ahunaunaitī- gāala

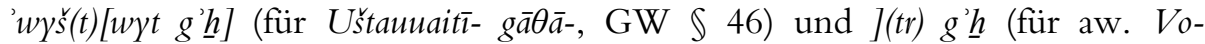
huxša $a$ ra- gā $\theta \bar{a}-,, G W \ 65)$ belegt. Diese manichäische Einverleibung awestischer Bezeichnungen verwundert nicht; unklar ist, ob nask auch ein manichäisches Werk bezeichnen konnte, aber GW \$21 scheint dies zuzulassen.

SUNDERMANN 1984a, S. 227 Anm. 2 weist ausdrücklich darauf hin, dass die in der zoroastrischen Literatur verwendeten Textbezeichnungen wie z.B. dar 'Kapitel', wizār 'Traktat', dādestān 'Urteil', wizìdagīhā 'Auswahl', kārnāmag 'Chronik' und ayāidgār 'Gedenkschrift' keine Verwendung bei den Manichäern fänden.

\section{Bezeichnungen von Textteilen}

\section{1 hnd'm}

Wie bereits erwähnt, nannten WALDSCHMIDT-LENTZ die parthischen Hymnenzyklen 'Gliedhymnen' nach dem in Überschriften und in Rubriken vorkommenden Wort hnd' $m$ 'Gliedmaß'. Für dieses Wort in der Verwendung als literarischer Terminus wird oft 'canto' als Übersetzung gewählt, da mit ihm auch ein umfangreicher Teil eines noch längeren Texts bezeichnet werden kann. Auf die Art der Vorführung des Verstextes hebt das Wort hnd'm nicht ab. Obwohl in der Form mittelpersisch, wird es in Bezug auf parthische Texte verwendet. Die echt parthische Form des Wortes ist einmal direkt ('nd' $m$ LN \ 21) und mehrmals als Lehnwort im Sogdischen ' $n \delta$ ' $m y$ belegt.

\subsection{Gwy̌sn}

Für mittelpersisch gwyšn gibt SUNDERMANN 1986, S. 66 die Bedeutungen 'Abschnitt von Lehrschriften, Predigt, Traktat, Versrede'. SUNDERMANN 
1984a, S. 228 stellte folgende Verwendungen von gwyšn fest: 1. Abschnitte von Lehrschriften: 'Kapitel'; 2. Predigten: 'Rede, Homilie'; 3. Versreden: 'Rede'. S. saxwan für die syrische Vorlage mēmrā. Der Terminus ist also sowohl für ganze Texte als für Textteile belegt.

Wie bereits erwähnt, kommt gōwǐ̌n im Titel des mittelpersischen Werks Gōwišn i grīw zindag vor. Das Wort ist ebenfalls in Kapitelüberschriften belegt. Bei beiden hat man die Wahl zwischen den Bedeutungen 'Rede' = 'Aussprache' oder 'Kapitel' etwa als Teil einer Zeremonie. In dem mittelpersischen Werk redet die Lebendige Seele, also passt die Bedeutung 'Rede'. Das Wort wäre nur dann ein Terminus, wenn eine solche literarische Rede eine Standardform mit dieser Bezeichnung geworden wäre. Gewissermaßen sind die zwei parthischen Hymnenzyklen ebenfalls Klagereden der Seele eines Verstorbenen, aber die literarische Bezeichnung für diese Werke kennen wir nicht, denn sie werden mit ihren Titeln bezeichnet. Die kleine Anzahl von Dialoghymnen scheint auch keine besondere Bezeichnung zu haben (z.B. die Überschrift in S 9, auf dem der Dialoghymnus mit dem Knaben Jesus zu finden ist, hat nur mhr'n 'gr'w'n 'vornehme Hymnen').

Die Bezeichnung gwy šn 'br im mittelpersischen kosmogonischen Text z.B. in der Überschrift hnzpt gwyšn 'br 'stwnd 'Vollendet ist: Die Erörterung über das Stoffliche' zeigt gwyšn als Kapitelbezeichnung, für die sich die Übersetzung 'Erörterung' eignet. Dies kann auch für gwy̌̌n mit einem folgenden ' $y$ gelten z.B. M7984/II/V/ii/9-10/ ${ }^{\circ} n w y s t$ gwyy $(\tilde{s})[n]{ }^{\circ} \circ$ 'y nrys $\underline{h} y z d$ ' 'Angefangen hat: die Rede/die Erörterung/das Kapitel über Gott Narisah', obwohl in einem anderen Kontext 'die Rede Gott Narisahs' hätte gemeint sein können.

In M172/I/V/10ff/ gwyšn 'y 'wnglywn zyndg könnte gwyšn 'das Sagen' bedeuten - MACKENZIE übersetzt 'The saying of - im Sinne von 'Sprechen, Vortragen'. Da die sogdische Übersetzung dieses Satzes fehlt, kann die Richtigkeit dieser Vermutung nicht überprüft werden.

Die gewählte Übersetzung 'Erörterung' als Alternative zu der Bedeutung 'Kapitel' könnte dann gelten, wenn $g w y$ šn als Kapitelbezeichnung auf vorgelesene Bücher oder sogar auf einen im Buch reproduzierten mündlichen Beitrag Manis (ähnlich den koptischen Kephalaia) hinweisen sollte. 'Diskurs' wäre gleichfalls eine mögliche Übersetzung.

S1/V/7-8/ 'br gwy̌sn ' $y d w$ 'zdh sxwn: 'Über das Sagen/Vortragen der 12 Reden/Ansprachen.'

Viele Rubriken haben die einfache Form 'br [...] 'über [...]'. Unklar ist, ob gwy̌̌n immer mit zu verstehen ist. Im Alternativtitel 'br hry 'yr wyfr's 'der Sermon von den drei Dingen' zum gy'n wyfr's 'der Sermon der (= von der) Seele' ist 'br von wyfr's abhängig. 


\subsection{Parabeln und ihre Ausdeutung}

'zynd 'Erzählung'. SUNDERMANN 1984a, S. 237 stellt einen etymologischen Zusammenhang mit zand fest. Dies ermöglicht es, vielleicht eine Nuance 'Auslegung' neben 'Erzählung' festzustellen. Auf S. 238 merkt er an, dass christlichsogdisch 'znt das syr. taš 'ịt $\bar{a}$ 'narratio' oder syr. šarba 'narratio, historia, sermo, tractatus, quaestio, thema, res, causa' übersetze. In Texten über Manis Missionstätigkeit, d.h. vor allem in denen, die seine Auseinandersetzungen mit hochrangigen Personen schildern, wird das gezielte Einsetzen von Erzählstoffen dargestellt, z.B.

parthisch MKG(81-84) 'wt frystg 'w twr'n šs' $\underline{y}$ 'zynd w'xt ${ }^{\circ \circ}$ mrd ' $y w$ ' $h$ 'z (') $[w] d$ hft pwhr bwd ${ }^{\circ}$

'Und der Apostel erzählte dem Tūrān-šāh ein Gleichnis: 'Es war (ahāz) ein Mann und (er) hatte sieben Söhne." (nach SundERMANN 1981, S. 22).

Auf Grund der eindeutigen Übereinstimmung mit den Gleichnissen des Neuen Testaments werden hier die Bezeichnungen 'Gleichnis' und 'Parabel' verwendet. Die Ausdeutung der Geschichte (auch 'Epimythion' genannt) erfolgt oft sehr knapp, z.B.

$\operatorname{KPT}(1721) w c^{\prime} r y \check{s}^{\circ} \circ\left({ }^{\prime}\right) k w h$ myrd nywšs g:(n) (hyn)d

'Die Deutung: Der niedriggeborene Mann, das sind die Hörer, [...]'

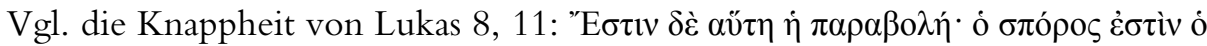
$\lambda$ óyos toṽ $\theta \varepsilon o \tilde{v}$. Allerdings verwendet der griechische Text keinen Terminus für die Ausdeutung der Parabel.

Im Sogdischen wird ' $z$ 'nd ebenfalls verwendet, (z.B. in der Parabelrolle, die die Überschrift " $z$ 'ntn'my 'Buch der Erzählungen' trägt). Dazu kommt das Wort prz $\beta r$ 'Parabel'.

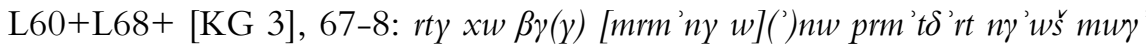
' $y w$ prz- $\beta r w^{\prime} \beta$ 'n $k t$ ' $y w$ my $(\delta)$

'And Lord Mar Mani said: 'Listen, magus, let me tell (you) a parable: One day [...]” (SimS-Williams 1990, S. 285).

Für 'Ausdeutung' gibt es die Termini parthisch $w c y h y s ̌ n$, mittelpersisch $g w z$ 'r und sogd. $x w y c q$ ' $w \gamma, x w y c q$ 'wy $\underline{h}$ 'Öffnung, (allegorische) Deutung', z.B. Tale A $61 x w y c q$ 'wy $\delta \beta r^{\prime}$ nd kt 'sie geben als Ausdeutung: [...]'

Die Nähe zwischen 'Erzählung' und 'Erklärung' ist im folgenden sogdischen Zitat aus einem kirchengeschichtlichen Text deutlich sichtbar, in dem vielleicht das erste Wort einfach für beide verwendet wird:

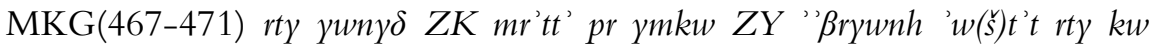

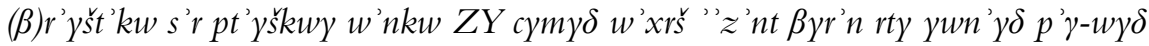
$Z Y^{\prime \prime} \gamma^{\prime}-t Z K \beta r^{\prime} y s^{\prime} t^{\prime} k r t s{ }^{\prime} y \beta r t p \delta y^{\prime} k h \delta^{\prime} \beta r \ldots$ 
'Und sogleich stand Mār Addā in Bitte und Gebet da, und zum Apostel sprach er: 'Die Erklärung ('z'nt) dieser ^Kunde möchte ich erlangen.' Und sogleich wurde enthüllt und kam der Apostel und gab ihm Wissen, [...]' (SUNDERMANN 1981, S. 43)

\subsection{Ist eine Bezeichnung für 'Text' belegt?}

Bedeutet das Wort nbyg nur 'Buch' oder möglicherweise auch 'Text'?

M16/V/8-9/ ('wd pd) '(n)yc zrdrwsttg'n nbyg ...

'und in noch einem anderen zoroastrischen Buch/Text [...]'

Wenn nbyg hier 'Buch' bedeutet, kann es durchaus auf ein Buch ab der Mitte der Sasanidenzeit hinweisen; wenn es 'Text' bedeutet, lässt es die Frage nach dem Zeitpunkt für die Verschriftlichung zoroastrischer Texte unbeantwortet.

Mittelpersisch ' $y n$ mhrn'mg (M1) ist die Bezeichnung des Textes, aber auch des Buchs, von dem M1 ein Teil ist.

Das Parabelbuch hat den sogdischen Titel 'z'ntn'my 'Parabelbuch', der wohl die Bezeichnung eines Buchs oder zumindest einer Sammlung mahnender Erzählungen ist. Aber es muss angemerkt werden, dass das ähnlich gebildete sogdische $n^{\prime} \beta n^{\prime} m^{\prime} k$ aus $n^{\prime} \beta$ 'Volk' und $n^{\prime} m^{\prime} k$ keineswegs die Bezeichnung eines Buchs ist; hier handelt es sich um eine Liste von Völkern auf einem einzigen losen Stück Papier.

Das Chinesische Compendium bestätigt wohl den Gebrauch des Wortes dēwān als Bezeichnung einer Sammlung von Manis Briefen (s. HALOUN/HENNING 1952, S. 207).

\subsection{Textbestandteile}

Parthisch $p d w^{\prime} g$ 'Refrain'

Das am Rand einer Handschriftenseite mit Verstext geschriebene $<\mathrm{p}>$ (auf der Rectoseite steht der Buchstabe Kopf zum Text, auf der Versoseite steht er in derselben Orientierung wie der Text) ist schon früh als parth. $p d w^{\prime} g$ 'Erwiderung, Responsium, Refrain' gedeutet worden.

Sogdisch $w^{\prime} x x_{s}^{\prime}$ 'Strophe'

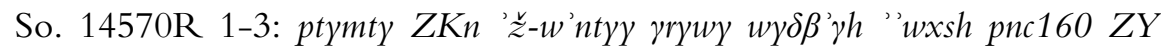
' $y w$ w'x $x$ š

'Beendet: der Lebendigen Seele, Erörterung, circa 560 und 1 Strophen.'

SUNDERMANN 1984a, S. 230 Anm. 23 hält dies für eine spezielle Verwendung von $w^{\prime} x s^{r}$ 'Wort'.

Mittelpersisch $g \gamma^{\prime} g$ 'Ort' hat auch die Bedeutung: 'Stelle in einem Text' 
M9/I/V/15/ 'ndr 'yn nbyg pd ws gy'g 'in diesem Buch an vielen Stellen' Die Präposition 'c hat in Bezug auf Texte die Bedeutung 'Teil aus/von'.

\section{Zusatz (andarz)}

Als Bezeichnung der bekannten Gattung der zoroastrischen Weisheitsliteratur im Mittelpersischen, wo mehrere Texte dieses Wort im Titel enthalten, ist das relativ häufig in der manichäischen Literatur belegte Wort ' $n d r z$ auch einen Blick wert. Es bedeutet vor allem 'Anweisung, Gebot'. Für den Beleg M18/R/6/ kw ' $y m r^{\prime} z$ 'ndrz d'ryd hat SUNDERMANN die Übersetzungen 'bewahrt dieses Geheimnis' und 'respektiert dieses Geheimnis' vorgeschlagen. Für eine ähnliche, aber teilweise ergänzte Formel M763/V/ii/20/ ql'n 'nd(rz) [d'ryd] hat RECK die Übersetzung 'das reine Gebot [haltet]' vorgeschlagen. Für M5561+M5562/R/1/ [•••••••] 'ndrz d'ryd ${ }^{\circ}$ wählt SUNDERMANN einfach '[...] er bewahrt'. (Kommentar SUndERMANN 1997, S. 123 GW \ 70, 1). Es gibt keinen Hinweis auf eine Verwendung dieses Worts in der in zoroastrischen Texten belegten Weise. Dennoch s. DE BLOIS 1984 und YOSHIDA 2001, S. 109 für einen Beleg für die manichäische Verwendung eines zoroastrischen andarz-Stoffes.

YOSHIDA 2001, S. 110f. zeigt, dass die manichäische Literatur den Terminus $y w k$ 'Lehre' für andarz-artige Anweisungen verwendet.

\section{Abkürzungen}

Ac. Ir. Acta Iranica

APAW Abhandlungen der Preußischen Akademie der Wissenschaften

BSOAS Bulletin of the School of Oriental and African Studies

BT Berliner Turfantexte

GW S. SUNDERMANN 1997

JRAS Journal of the Royal Asiatic Society

KPT S. SUNDERMANN 1973

LN s. SUNDERMANN 1992

MKG s. SUNDERMANN 1981

ZorMP zoroastrisches Mittelpersisch

\section{Bibliographie}

Boyce, MARy (1960): A Catalogue of the Iranian Manuscripts in Manichean Script in the German Turfan Collection, Berlin (Deutsche Akademie der Wissenschaften zu Berlin, Institut für Orientforschung, Veröffentlichung Nr. 45). 
Boyce, Mary (1968): Middle Persian Literature. In: Bertold Spuler (Hrsg.), Handbuch der Orientalistik, Iranistik II, 1, Leiden/Köln, S. 31-66.

DE Blois, FRAnçOIS (1984): The admonitions of Ādurbād and their relationship to the Ahīqar legend. In: JRAS, S. 41-53.

Durkin-Meisterernst, Desmond / EnRiCo Morano (2010): Mani's Psalms. Middle Persian, Parthian and Sogdian fragments in the Turfan Collection, Turnhout (BT XXVII).

GARDNER, IAIN (2011): Manichaean ritual practicse at ancient Kellis: A new understanding of the meaning and function of the so-called 'prayer of the emanations', in: Jacob Albert van Den Berg et al. (Hrsg.): In Search of Truth: Augustine, Manichaeism and other Gnosticism. Studies for Johannes van Oort at Sixty. Leiden u.a. (Nag Hammadi and Manichaean Studies 74), S. 245-262.

Haloun, Gustav / Walter Henning (1952): The Compendium of the Doctrines and Styles of the Teaching of Mani, the Buddha of Light. In: Asia Major N.S. 3, S. 184-212.

HenNing, WALter BRUno (1936): Ein manichäisches Bet- und Beichtbuch, $A P A W$ 1936, 10, Berlin 1937 [= Selected Papers I, S. 417-558].

Henning, Walter Bruno (1940): Sogdica, London (James G.G. Forlong Fund, XXI) [= Selected Papers II, S. 1-68]. Errata sheet with some addenda to Sogdica, 1941.

Henning, Walter Bruno (1944): The Murder of the Magi. In: JRAS, S. 133-149 [= Selected Papers II, S. 139-150].

HutTER, MANFRED (1992): Manis kosmogonische Šäbuhragān-Texte, Wiesbaden (Studies in Oriental Religions 21).

MacKenzie, David Neil (1994): »I, Mani .. «. In: Holger Preissler / Hubert SEIWERT (Hrsg.), Gnosisforschung und Religionsgeschichte. Festschrift Rudolph. Marburg, S. 183-198.

Mikkelsen, GunNer (2006): Dictionary of Manichaean Texts in Chinese, Turnhout (Corpus Fontium Manichaeorum. Dictionary of Manichaean texts. Vol III. Texts from Central Asia and China. Part 4).

MORANO, ENRICO (2009): "If they had lived ..." A Sogdian-Parthian Fragment of Mani's Book of Giants. In: Werner Sundermann / Almut Hintze / FRANÇOIS DE BloIS (Hrsg.), Exegisti monumenta. Festschrift N. Sims-Williams, Wiesbaden (Iranica 17), S. 325-330.

Moriyasu, Takao (2011): The Discovery of Manichaean Paintings in Japan and Their Historical Background. In: JACOB AlBERT VAN DEN BERG et al. (Hrsg.), In Search of Truth: Augustine, Manichaeism and Other Gnosticism. Studies for Johannes van Oort at Sixty. Leiden u.a. (Nag Hammadi and Manichaean Studies 74), S. 339360 .

MÜller, Friedrich Wilhelm KARL (1904): Handschriften-Reste in Estrangelo-Schrift aus Turfan, Chinesisch-Turkestan. II. Teil, Berlin (APAW 1904, Anhang Nr. 2).

ReCK, Christiane (2004): Gesegnet sei dieser Tag. Manichäische Festtagshymnen. Edition der mittelpersischen und parthischen Sonntags-, Montags- und Bemahymnen, Turnhout (BT XXII). 
Sims-Williams, Nicholas (1983): Indian Elements in Parthian and Sogdian. In: Klaus RöHrborn / Wolfgang Veenker (Hrsg.), Sprachen des Buddhismus in Zentralasien. Vorträge des Hamburger Symposions vom 2. Juli bis 5. Juli 1981, Wiesbaden (Veröffentlichungen der Societas Uralo-Altaica 16), S. 132-141.

Sims-Williams, Nicholas (1990): The Sogdian fragments of Leningrad II: Mani at the court of the Shahanshah. In: Bulletin of the Asia Institute N.S. 4, 1990 [1992], S. 281-288.

SimS-Williams, Nicholas (1991): The Sogdian fragments of Leningrad III: Fragments of the Xwāstwānīft. In: Aloïs van TONGERloO / Søren Giversen (Hrsg.), Manichaica Selecta ... presented to J. Ries, Louvain (Manichaean Studies I), S. 323-328.

SUNDERMANN, WERNER (1973): Mittelpersische und parthische kosmogonische und Parabeltexte der Manichäer. Mit einigen Bemerkungen zu Motiven der Parabeltexte von Fr. GEISSLER, Berlin (BT IV).

SUNDERMANN, WERNER (1975): Überreste manichäischer Yimki-Homilien in mittelpersischer Sprache? In: Monumentum H.S. Nyberg, 2, Téhéran/Liège (Acta Iranica 5), S. 297-312 [= W. SUNDERMANN: Manichaica Iranica 2, S. 597-612 and 613-614].

SUNDERMANN, WeRnER (1981): Mitteliranische manichäische Texte kirchengeschichtlichen Inhalts mit einem Appendix von NichOlas SimS-WiLliams, Berlin (BT XI).

SUNDERMANN, WERNER (1984a): Die Prosaliteratur der iranischen Manichäer. In: WojCiech SKalmowski / Alö̈s VAN TONgerloo (Hrsg.), Middle Iranian Studies, Leuven, S. 227-241 [= W. SundermanN: Manichaica Iranica 1, S. 183197 und 198].

SundermanN, WeRneR (1984b): Ein weiteres Fragment aus Manis Gigantenbuch. In: Orientalia J. Duchesne-Guillemin emerito oblata, Leiden (Ac.Ir. 23), S. 491-505 [= W. SUNDERMANN: Manichaica Iranica 2, S. 615-629 and 630-631].

SUNDERMANN, WERNER (1986): Studien zur kirchengeschichtlichen Literatur der Manichäer I. In: Altorientalische Forschungen 13.1, S. 40-92 [= W. SUNDERMANN: Manichaica Iranica 1, S. 217-269].

SundermanN, Werner (1992): Der Sermon vom Licht-Nous. Eine Lehrschrift des östlichen Manichäismus. Edition der parthischen und sogdischen Version, Berlin (BT XVII).

SUNDERMANN, WERnER (1994): Mani's "Book of the Giants" and the Jewish Books of Enoch. A case of terminological difference and what it implies. In: SHAUL SHAKed / AMNON NetZer (Hrsg.), Irano-Judaica III, Jerusalem, S. 40-48 [= W. SUNDERMANN: Manichaica Iranica 2, S. 697-705 and 706].

Sundermann, Werner (1997): Der Sermon von der Seele. Eine Lehrschrift des östlichen Manichäismus. Edition der parthischen und soghdischen Version mit einem Anhang von PETER ZiemE: Die türkischen Fragmente des "Sermons von der Seele". Mit 98 Abb. auf 47 Taf., Turnhout (BT XIX).

SundermanN, Werner (2004): Zarathustra der Priester und Prophet in der Lehre der Manichäer. In: Michael Stausberg (Hrsg.), Zoroastrian rituals in context, Leiden/ Boston, S. 517-530. 
Sundermann, Werner (2005): Was the Ardhang Mani's Picture-Book? In: Aloïs VAN TONGerloo, Luigi Cirillo (Hrsg.), Il Manicheismo nuove prospettive delle richerca!!], Turnhout, S. 373-384.

Yoshida, YutAkA (1983): Manichaean Aramaic in the Chinese Hymnscroll. In: BSOAS 46, S. 326-331.

YoshidA, YutAKa (1986): Remarks on the Manichaean Middle Iranian terms transcribed in Chinese script (1). In: Studies on the Inner Asian Languages 2, S. 1-15.

Yoshida, Yutaka (2001): On the Sogdian fragments of the St. Petersburg collection. In: Contributions to the Studies of Eurasian Languages Series 3, S. 105-117.

YoshidA, Yutaka (2010): Cosmogony and church history depicted in the newly discovered Chinese Manichaean paints. In: Daiwa bunka 121, S. 1-34. 
